\title{
Laboreal
}

Volume $10 \mathrm{~N}^{\circ} 2$ | 2014

Análise ergonómica do trabalho e formação - Parte II

\section{El análisis del trabajo: longevidad y desarrollo de un concepto}

A análise do trabalho: longevidade e desenvolvimento de um conceito

L'analyse du travail: longévité et développement d'un concept.

Work analysis: longevity and development of a concept

\section{Anne Lancry-Hoestlandt}

\section{CpenEdition}

Journals

Edición electrónica

URL: http://journals.openedition.org/laboreal/5026

DOI: $10.4000 /$ laboreal.5026

ISSN: 1646-5237

\section{Editor}

Universidade do Porto

\section{Referencia electrónica}

Anne Lancry-Hoestlandt, «El análisis del trabajo: longevidad y desarrollo de un concepto », Laboreal [En línea], Volume 10 N² | 2014, Publicado el 01 diciembre 2014, consultado el 08 octubre 2019. URL : http://journals.openedition.org/laboreal/5026 ; DOI : 10.4000/laboreal.5026

Este documento fue generado automáticamente el 8 octubre 2019.

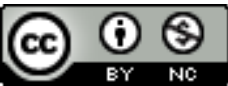

Laboreal está licenciado com uma Licença Creative Commons - Atribuição-NãoComercial 4.0 Internacional. 


\title{
El análisis del trabajo: longevidad y desarrollo de un concepto
}

\author{
A análise do trabalho: longevidade e desenvolvimento de um conceito \\ L'analyse du travail: longévité et développement d'un concept. \\ Work analysis: longevity and development of a concept
}

Anne Lancry-Hoestlandt

\section{REFERENCIA}

Karnas, G., \& Salengros, P (1986). L'analyse du travail trente ans après Ombredane et Faverge. In R. Patesson. L'Homme et l'écran. Aspects de l'ergonomie en informatique (p. 331-340). Colloque "L'ergonomie en informatique", 1985, Nivelles. Bruxelles: Ed.de l'Université de Bruxelles.

\section{NOTA DEL EDITOR}

http://dx.doi.org/10.15667/laborealx0214ac

Este texto de Guy Karnas y Pierre Salengros [ ${ }^{1}$ ] es una ponencia presentada en el Coloquio "Ergonomía e informática" organizado en Nivelles, Bélgica, en noviembre 1985 por René Patesson y el grupo de Investigaciones en Informática y Ciencias Humana de la Universidad Libre de Bruselas (1986). En aquel entonces investigadores del laboratorio dirigido por Jean-Marie Faverge, quien había estado implicado en las primeras investigaciones europeas en el marco de la Comunidad Europea del Carbón y del Acero (CECA), Karnas y Salengros desarrollaron la práctica del análisis del trabajo, tanto como método que como objeto, en una gran variedad de puestos y situaciones de trabajo. Dichos investigadores precisan en este artículo "que éste (el análisis del trabajo) solo se puede concebir refiriéndose a un objetivo con respecto al cual el mismo es 'método'" (p. 332, traducción libre). Esta línea de investigación y de prácticas cuyo 
origen se encuentra en los trabajos de Ombredane y Faverge, surge en el contexto de los años 50 y 60 en el cual el trabajo es sobre todo físico y en donde la transmisión de señales informativas son relativamente poco simbolizados. Es sumamente interesante poder constatar y recordar que esa línea de investigación está muy presente desde los años 80 en sectores de actividad diversos cuyo desarrollo tecnológico, en expansión rápida y constante, transformó y modificó radicalmente casi la totalidad de las situaciones de trabajo.

2 El propósito de los autores es mostrar que 30 años después de la publicación de la obra de referencia de Ombredane y Faverge (1955), los principios fundadores del análisis del trabajo son lo suficientemente robustos y evolutivos permitiendo así el análisis de situaciones de trabajo completamente nuevas y desconocidas en los años 50. Ahora, casi 60 años después de la publicación de esta obra, los conceptos siguen siendo de actualidad y se apoyan en desarrollos más recientes adaptados a las evoluciones del trabajo. Es lo que mostró la jornada de estudios organizada por el GRESHTO-CRTD [2] del CNAM en 2012 dedicada al libro de André Ombredane y de Jean-Marie Faverge publicado en 1955 "L'analyse du travail. Facteur d'économie humaine et de productivité" [3].

3 Faverge, retomado por Karnas y Salengros, constata que el orden cronológico de la aparición de los análisis del trabajo coincide con la evolución de las modificaciones tecnológicas y ergonómicas del trabajo. Citemos el texto original: “- trabajar es adoptar posturas, realizar gestos; - trabajar es procesar información, es comunicar con la materia, ya sea directamente, ya sea mediante la ayuda de intermediarios más o menos complejos, desde el dial, el botón, el pedal, hasta la computadora o el robot, pasando por el autómata; - trabajar es también regular procesos complejos como resultado de las interacciones entre las células de un sistema del cual uno mismo es uno de los elementos que - y esto es evidente - es también un sistema; trabajar es finalmente emplear algoritmos, heurísticos, representaciones, estrategias. Es allí el ámbito de lo que se convirtió en la ergonomía cognitiva" (p. 332 y 333, traducción libre).

4 En el marco del coloquio sobre la Ergonomía Informática, los autores ponen en relieve tres características principales que deben ser tomadas en cuenta en el análisis del trabajo: “- la apropiación del Hombre sobre su actividad, los movimientos de humanización del trabajo, y el desarrollo de las técnicas informáticas en el ámbito de una crisis económica con sus consecuencias sobre la dinámica organizacional de la empresa" (p. 333, traducción libre). La previsión (por el operador y no únicamente por la oficina de ingeniería), la regulación de la actividad, la posibilidad de intervenir sobre una cadena de disfuncionamientos (Faverge et al., 1966) son elementos que corroboran el interés y las habilidades de trabajo desarrolladas, y de la apropiación del Hombre sobre su actividad; son por lo tanto aspectos importantes que se deben considerar en el análisis del trabajo. Relacionamos estas observaciones con los trabajos más recientes de Clot (2013) quien, acerca de una reflexión sobre la obra de Malrieu, señala que "un trabajador no puede vivir durablemente en un oficio más que si él puede fabricar un oficio para trabajar (...) el oficio no es solo una actividad personal o interpersonal. Es también transpersonal e impersonal, es historia colectiva y "genérica" de un contexto, y normas sociales generales y prescritas de una profesión” (p. 148, traducción libre del traductor).

5 Regresando al texto de 1986 los autores señalan en un segundo punto que, conjuntamente con el desarrollo del análisis del trabajo en una perspectiva 
estrictamente ergonómica, más centrada en el puesto de trabajo in situ, algunas líneas de investigación en psicología social, sociología, economía se han interesado en los contextos de vida en los cuales el trabajador se desarrolla. Los autores se refieren a estudios relativos a las relaciones entre la vida profesional y la vida personal, en los cuales abordan las cuestiones sobre las representaciones del trabajo para el Hombre y los sistemas de valores relacionados. Al evocar los conflictos temporales entre vida profesional y vida personal, estos señalan las reinterpretaciones posibles de los umbrales de declaraciones de accidentes laborales o de los factores que inciden el ausentismo, y surge así la noción de "estilo", que refleja la manera en que cada uno percibe y regula su vida profesional. Esa inversión de ángulo desde el cual la ergonomía aborda el trabajo (la persona habla por ella misma) lleva a inevitablemente el empleo de otros métodos de investigación y de análisis, i.e. la entrevista y la interrogación.

Esta evolución permitió a los ergónomos enriquecer sus análisis con puntos de vista psico-sociales y trabajar con psicólogos sociales y psicólogos del trabajo. Hacemos particular alusión aquí al sistema de actividades de la vida profesional y personal, de los trabajos llevados a cabo por Curie (2000) en el laboratorio "Personnalisation et changements sociaux" (personalización y cambios sociales) de Toulouse.

7 En el tercer y último punto de su presentación, los autores abordan el desarrollo de las técnicas informáticas en un contexto de crisis económica. En los años 80 , se habla de puestos dedicados a la entrada de datos, que se acompañaban de un sentimiento de pérdida de autonomía y de libertad en la aplicación de los procedimientos, incluso un sentimiento de despersonalización en la relación con los clientes. Los primeros estudios en ese campo concierne la adaptación a la máquina y comienzan a aparecer estudios sobre los aspectos psicosociológicos de ese tipo de trabajo, los cuales van a asociar a sus cuestionamientos, un análisis del trabajo. Lo que permitirá en particular mostrar, por ejemplo, que la famosa "resistencia al cambio" no se le puede atribuir a una falta de motivación sino más bien a una pérdida de la posibilidad de anticipar la tarea, de controlar su actividad y de ser necesario, recuperar una situación errónea.

8 Sin entrar en detalles, esos estudios corresponden también al periodo en el cual Leplat (1992) y Leplat y Hoc (1983) retoman, con el fin de precisar, la distinción entre análisis de la tarea, descriptiva y diagnostica (prescrita, efectiva y real) y el análisis de la actividad en sus aspectos observables y en sus mecanismos subyacentes, inobservables y pudiéndose inferir. Numerosas técnicas permitiendo objetivar la tarea están disponibles y son a menudo necesarias para poner en obra el análisis de la actividad para el cual, además de la observación filmada, de las entrevistas y cuestionarios, se le pueden asociar los métodos de la psicología clínica de la actividad (auto confrontación simple, cruzada y método del "sosias" (Clot, 2008).

9 El enriquecimiento del análisis del trabajo y del análisis de la actividad acompaña por un lado el desarrollo de las nuevas formas de trabajo y, por otro lado, las reflexiones importantes sobre los sectores de actividades antiguas cuya evolución y disfuncionamientos plantean una serie de interrogantes. En ergonomía se han desarrollado nuevas temáticas que muestran que es posible transponer el análisis del trabajo a situaciones que se podía pensar a priori que no entraban dentro de su campo de aplicación (campos como de la ergonomía y la educación, de la ergonomía y del multimedia, de la salud, de la discapacidad, de los transportes, de los servicios y de la ergonomía escolar (Lancry, 2009). 
El desarrollo del análisis del trabajo en el medio escolar (Lancry-Hoestlandt, 2013, 2014) es otro ejemplo. Tiene como punto de partida los conceptos de tarea y de actividad especificándolos y enriqueciéndolos en especial con las nociones de prescripción explicita e implícita, de tarea principal (la que se evalúa) y de tarea secundaria (prerrequisito a la comprensión y a la realización de la tarea principal). Este enfoque permite también abordar la noción de fiabilidad retomando la concepción de Faverge del Hombre como agente de fiabilidad y de "infiabilidad" para él mismo y para el sistema, de allí que incluimos la definición de Leplat y de Terssac (1990): "Estudio de los factores (modalidades de implementación y de elaboración de competencias) propias a la mejora de la calidad del acoplamiento Hombres X tareas" (traducción libre del traductor). En el medio escolar se trata de identificar los factores, o la combinación de factores, que impiden o complican la realización de la tarea prescrita, o de las tareas secundarias. Se habla de indicadores de la fiabilidad cuando el actor (por ej. el alumno, el profesor u otro trabajador) puede acentuar o provocar una situación no fiable para su propia acción, o inversamente aportar elementos correctores que optimizan la situación e inhiben los efectos negativos que generan los errores o disfuncionamientos, i.e. cuando se vuelve agente de fiabilidad para el mismo y para la estructura. Estos primeros enfoques deben ser explorados y completados por un análisis conjunto de la actividad del alumno y del profesor.

Queda entonces claro que es útil y de gran valor poder retomar y "volver a hacer hablar" los textos e investigaciones fundadoras de nuestras disciplinas y constatar que les actualizaciones son posibles y fecundas.

\section{BIBLIOGRAFÍA}

Clot, Y. (2008). Travail et pouvoir d'agir. Paris : PUF.

Clot, Y. (2013). Philippe Malrieu et le travail : une personnalisation paralysée ? In A. BaubionBroye, R. Dupuy \& Y. Prêteur. (Eds). Penser la socialisation en psychologie. Actualité de l'œuvre de Philippe Malrieu (pp. 141-152). Toulouse: Erès.

Curie, J. (2000). Travail, Personnalisation, Changements sociaux. Archives pour les histoires de la psychologie du travail. Toulouse: Octarès.

Faverge,J-M., Olivier, M., Delahaut, J., Stephaneck, P., \& Falmagne, J.C. (1966).

L'ergonomie des processus industriels. Bruxelles: Editions de l'Institut de Sociologie de l'Université Libre de Bruxelles.

Lancry, A. (2009). L'ergonomie. Paris : PUF, Coll. Que sais-je?

Lancry-Hoestlandt, A. (2013). A propos d'ergonomie scolaire. In A. Drouin, (coord.). Ergonomie. Travail, Conception, Santé. Cinquantenaire de la Société d'Ergonomie de Langue Française - 1963-2013. (pp. 381-391).Toulouse: Octarès. 
Lancry-Hoestlandt, A. (à paraître 2014). Le travail en herbe. L'analyse du travail en situation scolaire. In R. Ouvrier-Bonnaz \& A. Weill-Fassina (coord.). "L'analyse du travail " Ruptures et Evolutions. Toulouse: Octarès (édition électronique en accès libre).

Leplat, J. (coord). (1992). L'analyse du travail en psychologie ergonomique. Recueil de textes. Tome 1. Toulouse: Octarès.

Leplat, J., \& Hoc, J. M. (1983). Tâche et activité dans l'analyse psychologique des situations. Cahiers de psychologie cognitive, 3, 1, 49-63.

Leplat, J. \& de Terssac, G. (1990). Les facteurs humains de la fiabilité dans les systèmes complexes. Toulouse: Octarès.

Ombredane, A. \& Faverge, J-M. (1955). L'analyse du travail. Paris : PUF.

Patesson, R. (coord) (1986). L'Homme et l'écran. Apports de l'ergonomie en informatique. Bruxelles : Editions de l'Université Libre de Bruxelles.

\section{NOTAS}

1.

[1] Karnas, G., \& Salengros, P (1986). L'analyse du travail trente ans après Ombredane et Faverge. In R. Patesson. L'Homme et l'écran. Aspects de l'ergonomie en informatique (p.331-340). Colloque "L'ergonomie en informatique", 1985, Nivelles. Bruxelles: Ed.de l'Université de Bruxelles.

2. Groupe de Recherche et d'Etude Sur l'Histoire du Travail et de l'Orientation du Centre de Recherche sur le Travail et le Développement du Conservatoire National des Arts et Métiers.

3. Esta Jornada dió origen a una obra dirigida por R. Ouvrier-Bonnaz y A. Weill-Fassina, del GRESHTO-CRTD, CNAM (se publicará en el 2014) : "L'analyse du travail" Ruptures et Evolutions. Ed. Octarès. Toulouse (edición electrónica en acceso libre).

\section{AUTOR}

\section{ANNE LANCRY-HOESTLANDT}

Conservatoire National des Arts et Métiers (CNAM), Centre de Recherche sur le Travail et le Développement (CRTD ), Groupe de Recherche et d'Etude sur l'Histoire du Travail et de l'Orientation (GRESHTO), 41 Rue Gay Lussac, 75005 Paris, France anne.lancry@cnam.fr 